\title{
Effect of sensor mounting and flow history on measured wall pressure spectra
}

\author{
$\mathrm{Nan} \mathrm{Hu}^{*}$ \\ German Aerospace Center (DLR), 38108 Braunschweig, Germany \\ Lars Erbig $^{\dagger}$ \\ Daimler AG, 71059 Sindelfingen, Germany
}

An experimental study of wall pressure fluctuations beneath a turbulent boundary layer was carried out on a plate model with a streamwise varying pressure gradient along the plate length. The wall pressure fluctuations were measured with flush-mounted Kulite sensors and mean velocity measurements across the boundary layer were performed by using a single-wire hot wire anemometer. A variety of streamwise pressure distributions were imposed by changing the position and the angle of attack of a NACA 0012 airfoil installed above the plate. The effect of the upstream flow history on the wall pressure spectra in the presence of the streamwise varying pressure gradient was studied. Spectral attenuation due to the spatial averaging associated with a finite sensor size was corrected via the Corcos correction. The results show that the Corcos correction to the measured Kulite spectra leads to an overprediction of the spectral intensity at medium frequencies and an underprediction at high frequencies. Hence, a new correction is proposed based on the measured spectral attenuation. Finally, the measurements also indicate that even slight imperfections in sensor mounting and relatively weak upstream flow disturbances can have a significant influence on the measured spectra of the wall pressure fluctuations.

\section{Nomenclature}

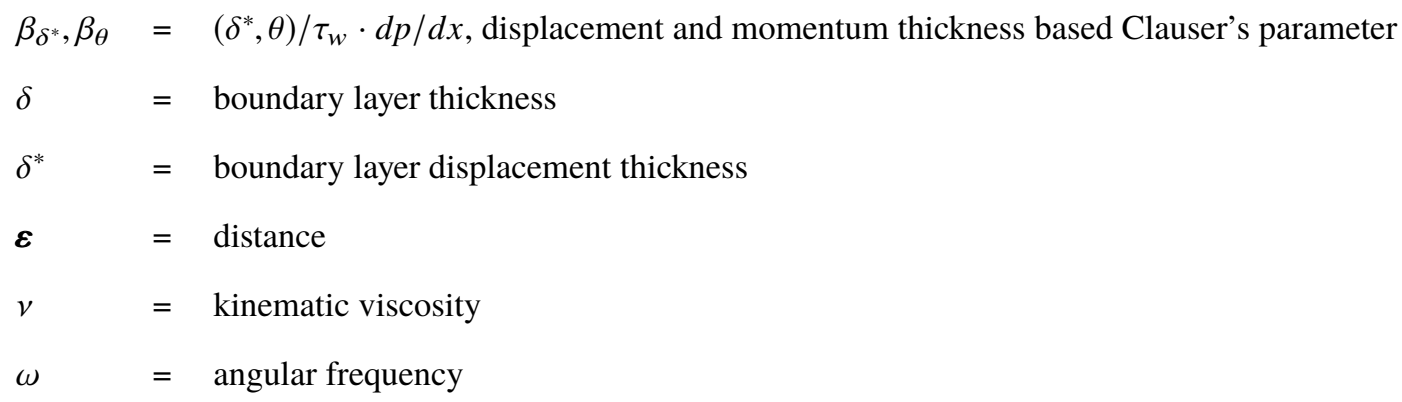

\footnotetext{
*Research engineer, Institute of Aerodynamics and Flow Technology, Department of Technical Acoustics, nan.hu@dlr.de ${ }^{\dagger} \mathrm{PhD}$ student, Aerodynamics- and Aeroacoustics Department
} 


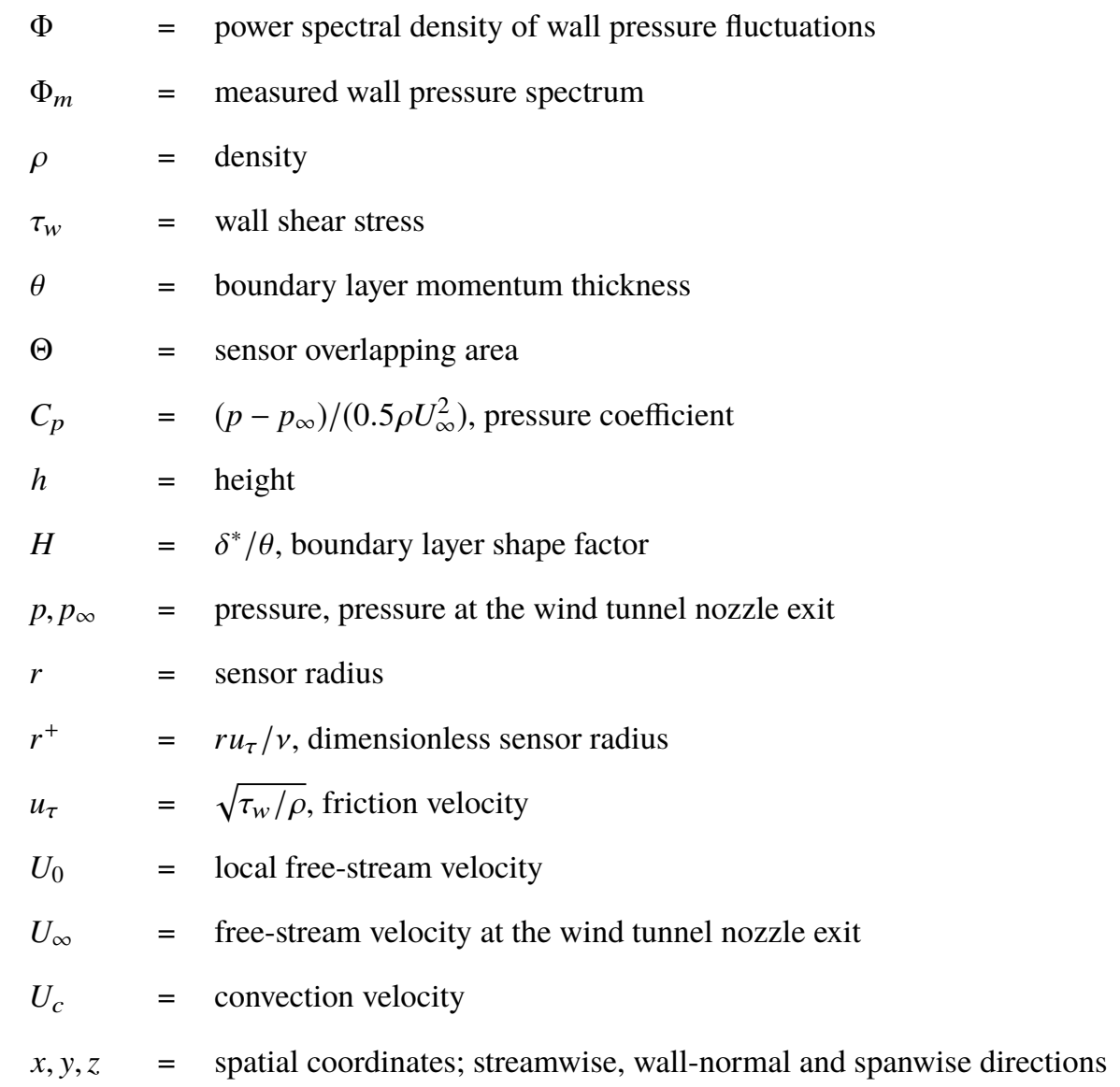

\section{Introduction}

Boundary layer induced wall pressure fluctuations are a fundamental topic in flow-induced noise. In early studies, most researchers focused on wall pressure fluctuations in zero pressure gradients (ZPG) [1-4]. However, a non-ZPG flow occurs in many practical situations. Recently, several studies [5-7] investigated the effect of a streamwise pressure gradient on the wall pressure fluctuations. Rozenberg et al. [8] proposed a wall pressure spectral model in adverse pressure gradients (APG) based on Goody's model [9], which is the most frequently used model in ZPG flows. Rozenberg argued that the effect of the pressure gradient on the wall pressure spectra could be evaluated correctly by Clauser's parameter. Later, Kamruzzaman et al. [10] and Catlett et al. [11] proposed the APG pressure models using a similar concept to Rozenberg's model. In contrast to those models, Hu [12] used the boundary layer shape factor to predict the spectral change due to the pressure gradient. In [12] it was pointed out that the local boundary layer parameters are predominantly determined by its upstream history, meaning that Clauser's parameter as a local pressure gradient based parameter could be an insufficient predictor of the wall pressure spectra in non-equilibrium flows.

It is challenging to accurately measure the wall pressure fluctuations at high frequencies. The difficulty arises from the signal averaging over the sensor surface. Typically, there is a choice of a flush-mounted construction or a 
pinhole-mounted construction. The attenuation of the wall pressure magnitude has to be measured at high frequencies due to the large ratio of the sensor size to the incident wavelength of hydrodynamic pressure fluctuations. This effect was first studied by Corcos [13], who established the relationship between the sensor size and its induced spectral attenuation based on modeled coherence of the wall pressure fluctuations. A uniform spatial sensitivity over the sensor surface was assumed in his study. Gilchrist \& Strawderman [14] and Farabee et al. [15] measured a non-uniform spatial sensitivity of actual sensors, which could significantly affect the accuracy of the attenuation proposed by Corcos. A pinhole-mounted construction could be used to reduce the effective sensor surface, thus, minimizing the spatial-averaging effect. However, the measurement frequency range, when employing a pinhole construction, will most likely limited by the Helmholtz resonance frequency.

Besides the drawback of the spatial-averaging effect for the flush-mounted construction, in practice, it is also difficult to achieve a perfect flush-mounting. The usual flush-mounting imperfections include small vertical offsets between a sensor surface and a wall, offsets due to surface curvatures as well as bore diameter tolerances. All those imperfections can perturb the local boundary layer flow and affect the measured wall pressure fluctuations.

In this work, the wall pressure fluctuations were measured on a plate model using flush-mounted Kulite sensors. Through displacement of the NACA 0012 airfoil installed above the plate, different streamwise pressure gradients on the plate boundary layer flow were produced. The experimental setup and the measurement techniques are described in Sec. II Results of the mean flow investigations are presented in Sec. III.A In Sec. III.B, the Corcos correction [13] is applied to the measured spectra to compensate for the high-frequency attenuation caused by the finite sensor size. Due to the insufficient agreement between the corrected spectra and the results from Hu \& Herr [7] which were measured with pinhole-mounted sensors in a similar experimental setup, a new correction for the high-frequency spectral attenuation is proposed. Furthermore, the effect of upstream flow history on the wall pressure spectra in the presence of a streamwise varying pressure gradient is investigated. In Sec. [II.C] the effect of installation-related disturbances on the measurements is evaluated by comparison with results for the disturbance-free configurations.

\section{Experimental Setup}

The experiment was conducted in the open-jet anechoic test section of the Acoustic Wind Tunnel Braunschweig (AWB). The wind tunnel has a rectangular nozzle with a height of $1200 \mathrm{~mm}$ and a width of $800 \mathrm{~mm}$. The maximum operating velocity is $65 \mathrm{~m} / \mathrm{s}$. Details of the experimental setup are documented in Fig. 11 A flat plate made from Ureol MB 600, was placed $10 \mathrm{~mm}$ downstream of the nozzle exit at the mid-height nozzle position. The plate surface was aligned with the flow direction. The length and thickness of the plate are $1775 \mathrm{~mm}$ and $80 \mathrm{~mm}$, respectively. The plate span is $1400 \mathrm{~mm}$, which is $300 \mathrm{~mm}$ wider than the nozzle exit on each side to prevent side-edge interaction with the AWB open-jet shear-layers, see Fig. 1 b). A $125 \mathrm{~mm}$ long super-elliptically shaped leading edge $\left(|x / 0.125|^{3}+|y / 0.04|^{3}=1\right.$ where $x$ and $y$ represent the streamwise and wall-normal directions) was used to avoid flow separation [16]. Both sides 
of the plate were tripped with $0.2 \mathrm{~mm}$ thick zigzag trip strips $100 \mathrm{~mm}$ behind the leading edge. A $12^{\circ}$ beveled trailing edge on the bottom side of the plate was used to realize a ZPG turbulent boundary layer in the rear area on the top side [17]. The trailing-edge tip was extended by foam serrations to minimize vortex shedding and to reduce trailing-edge noise.

Pressure gradients were produced by placing a NACA 0012 airfoil with $400 \mathrm{~mm}$ chord length and $1800 \mathrm{~mm}$ span width above the plate. The airfoil was installed $100 \mathrm{~mm}$ above the plate relative to the wing's chord at the geometric angle of attack (AOA) of $0^{\circ}$. This distance was fixed during the experiment. The AOA and the streamwise position of the airfoil were adjusted to produce the desired flow condition. The axis of rotation was at $41 \%$ of the chord length.

The static pressure along and across the plate was measured with 32 static pressure ports, see Fig. 1)(c). An L-shaped flush-mounted Kulite array was used to measure the wall pressure fluctuations. The Kulite sensor, model XT-140M-5PSI, has a diameter of $2.54 \mathrm{~mm}$ and is covered with a so-called B-screen (18 mini-holes around a $1.8 \mathrm{~mm}$ diameter circle). In the spanwise direction, the sensors were placed at $x=1165 \mathrm{~mm}(x=0$ for the leading edge $)$ with a constant separation of $8 \mathrm{~mm}$. The separations between the sensors in the streamwise direction were $12 \mathrm{~mm}, 10 \mathrm{~mm}$, and $8 \mathrm{~mm}$, respectively. A cavity with a $4 \mathrm{~mm}$ opening was mounted $35 \mathrm{~mm}$ downstream of the sensors to study gap noise excited by the boundary layer. The results of the gap noise study were discussed by Erbig et al. [18]. During the present test of the wall pressure fluctuations, the gap was covered with aluminum tape. The fluctuating pressures were recorded for $30 \mathrm{~s}$ with a sampling rate of $100 \mathrm{kHz}$ using a 16-bit GBM Viper data acquisition system. An external preamplifier (gain factor of 250) with a built-in first-order high-pass filter (cut-off frequency at $200 \mathrm{~Hz}$ ) was applied. The measured power spectra shown in this paper are corrected using the filter frequency response curve. The correction is applied down to $100 \mathrm{~Hz}$ at which the filter attenuation is $7 \mathrm{~dB}$. The attenuated spectral level at this frequency is much higher than the electrical noise level of the measurement system, which ensures the accuracy of the applied filter correction.

The mean flow velocities were measured by a Dantec single-sensor miniature wire probe, model 55P11. The hot-wire data were recorded for $20.4 \mathrm{~s}$ for each measurement point. A sampling rate of $50 \mathrm{kHz}$ and a low-pass filter with cut-off frequency at $20 \mathrm{kHz}$ were applied.

\section{Results}

\section{A. Mean flow characteristics}

The streamwise static pressure on the plate was measured between $865 \mathrm{~mm} \leq x \leq 1255 \mathrm{~mm}$. Fig. 22(a) shows the pressure coefficient $(C p)$ distributions for different flow conditions at a free-stream velocity of $U_{\infty}=30 \mathrm{~m} / \mathrm{s}$ at the nozzle exit. The APGs were produced by placing the airfoil at geometrical AOAs of $5^{\circ}$ and $8^{\circ}$. To achieve the desired local pressure gradient at the measurement position, the streamwise airfoil position was also varied by changing the parameter R. For example, R455 denotes $455 \mathrm{~mm}$ between the leading edge of the plate and the rotation axis of the 


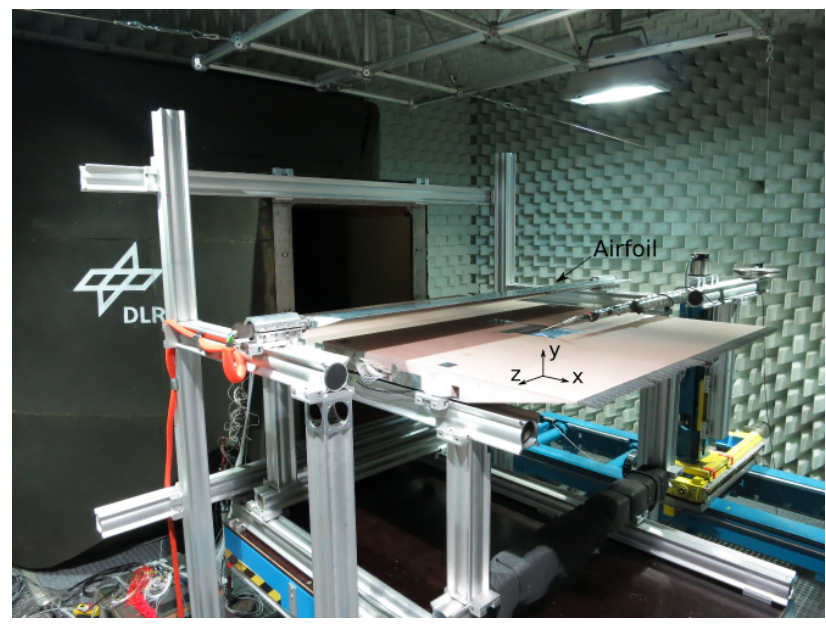

(a)

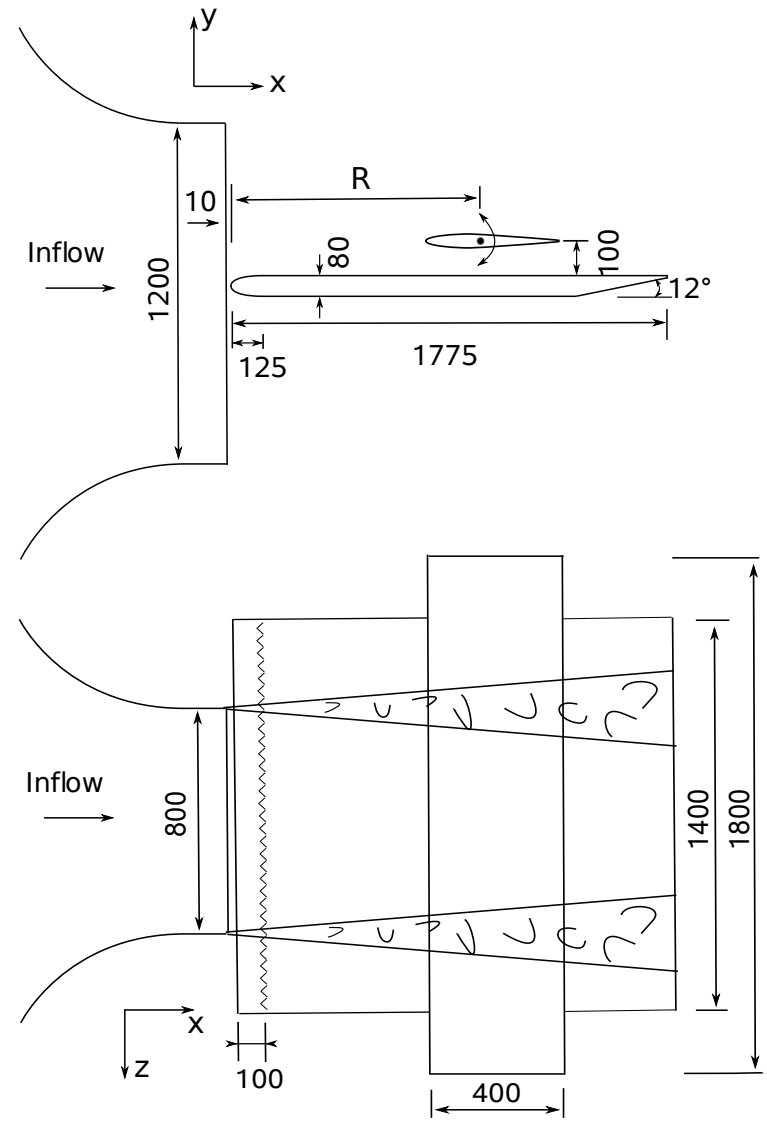

(b)

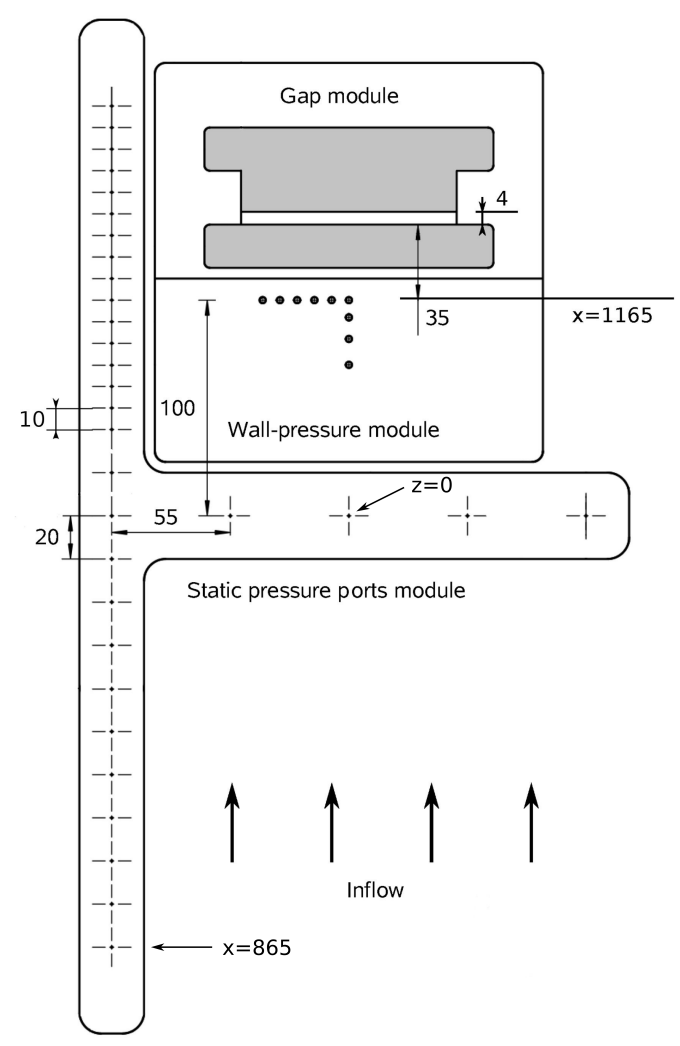

(c)

Fig. 1 (a) Photo of the experimental setup; (b) side- and top-view sketch; (c) Layout of Kulite sensors and static pressure ports. Dimensions are in mm. Numbering of the sensors: 1-4 in the streamwise direction from upstream to downstream; 4-9 in the spanwise direction from the mid-span to the side.

airfoil, refer to Fig. 11b).

The boundary layer mean velocity profile was measured at the location of the most downstream Kulite sensors, 


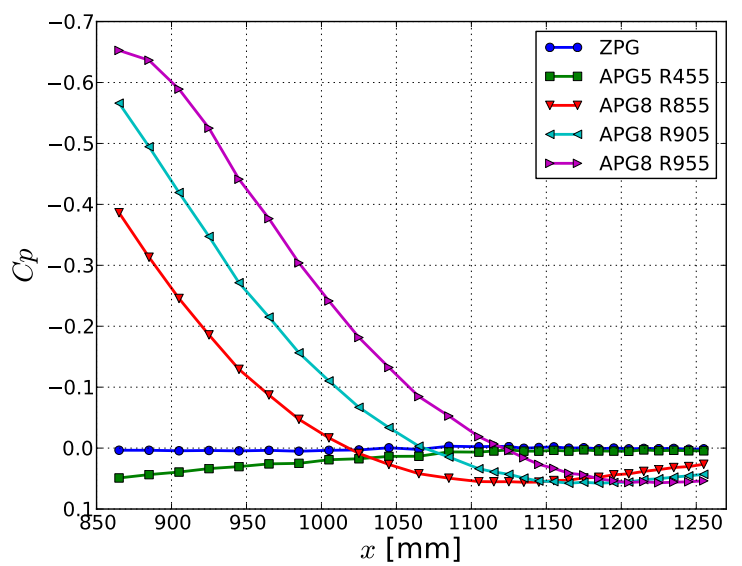

(a)

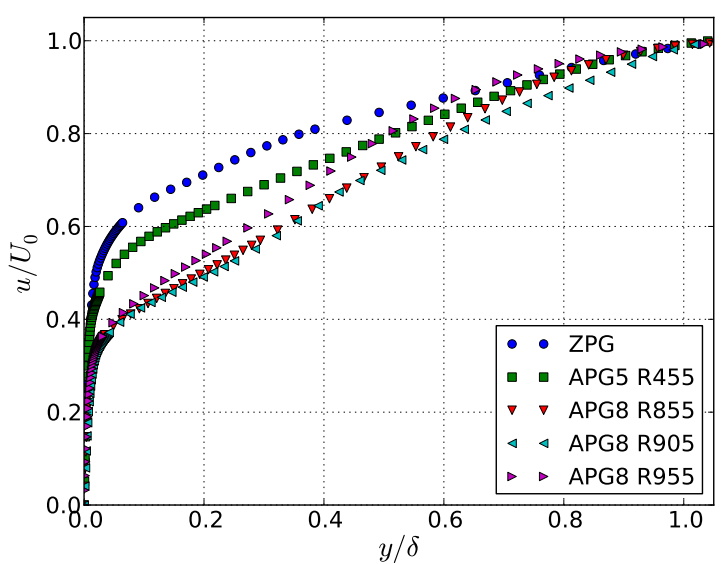

(b)

Fig. 2 (a) $C p$ distribution; (b) boundary layer profile at $x=1165 \mathrm{~mm}$.

Table 1 Boundary layer parameters.

\begin{tabular}{ccccccccc} 
& $U_{0}$ & $\delta$ & $\delta^{*}$ & $\theta$ & $H$ & $u_{\tau}$ & $d p / d x$ & $\beta_{\delta^{*}}$ \\
& $(\mathrm{~m} / \mathrm{s})$ & $(\mathrm{mm})$ & $(\mathrm{mm})$ & $(\mathrm{mm})$ & & $(\mathrm{m} / \mathrm{s})$ & $(\mathrm{Pa} / \mathrm{m})$ & \\
\hline ZPG & 31.4 & 18.7 & 3.35 & 2.4 & 1.39 & 1.16 & 34 & 0.07 \\
APG5 R455 & 32.2 & 36.5 & 8.16 & 5.43 & 1.5 & 0.95 & -35 & -0.27 \\
APG8 R855 & 30.6 & 34.7 & 10.03 & 5.68 & 1.77 & 0.73 & -120 & -1.9 \\
APG8 R905 & 30.4 & 28.7 & 8.68 & 4.92 & 1.76 & 0.74 & 13 & 0.17 \\
APG8 R955 & 30.6 & 28.9 & 7.36 & 4.28 & 1.72 & 0.75 & 270 & 2.97
\end{tabular}

$x=1165 \mathrm{~mm}$. Fig. 2(b) shows the measured profiles normalized with the boundary layer thickness $\delta$ and the local free-stream velocity $U_{0}$. The relevant boundary layer parameters are summarized in Table 1 . To determine the boundary layer displacement thickness $\delta^{*}$ and momentum thickness $\theta$, the mean velocities for locations $y<1 \mathrm{~mm}$ (not measured) are estimated using the Reichardt wall law [19]. The friction velocity $u_{\tau}$ is obtained by fitting the measurement data to the log-law region. The boundary layer profiles of the APG8 cases have a similar profile shape which shows a smaller velocity increase in the inner layer and a larger velocity increase in the outer layer when compared to the ZPG case. For these APG8 cases, they have a similar value of the boundary layer shape factor $H=\delta^{*} / \theta$, refer to Table 1 Similar to the shape factor, Clauser's parameter, defined as $\beta_{\delta^{*}}=\delta^{*} / \tau_{w} \cdot d p / d x$, is also an indicator of the boundary layer profile shape, which was developed and validated in equilibrium boundary layer flows [20-22]. However, different values of $\beta_{\delta^{*}}$ even with the opposite sign are obtained for the APG8 cases (non-equilibrium flows). These differences are caused by the different values of the local pressure gradient $d p / d x$.

The two-dimensional (2-D) flow condition was verified by ensuring a sufficiently weak spanwise variation in the mean flow velocity measured at different wall-normal locations, shown in Fig. 3. Due to the limitation of the operating distance of the traverse system, the velocity was only measured on one side from the mid-span $(z=0)$ outwards the 


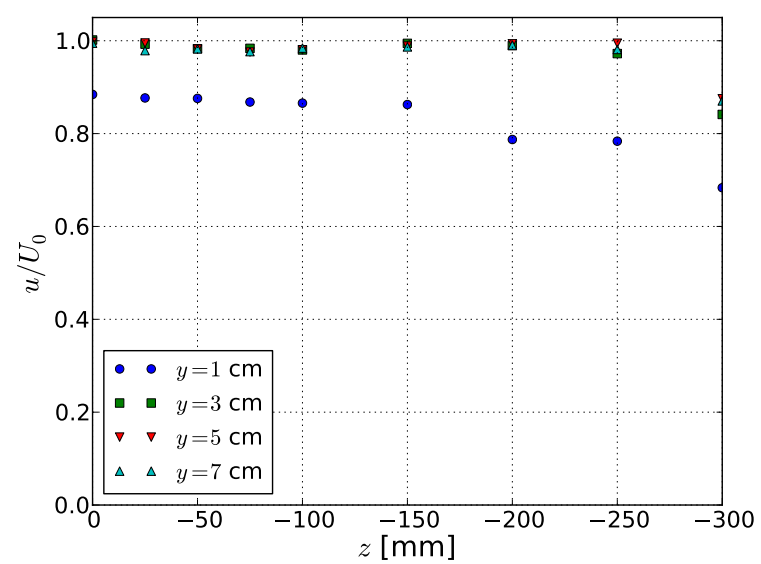

(a)

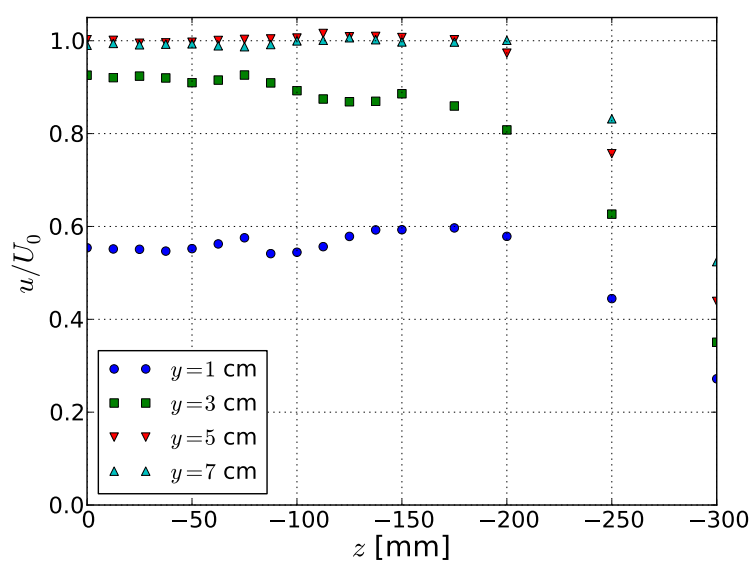

(b)

Fig. 3 The spanwise variation in the streamwise velocity component measured at selected wall-normal locations. (a) ZPG; (b) APG8 R855.

traverse system, refer to Fig. 1(a). The plate flow is assumed to be symmetric about the mid-span in the spanwise direction. Due to the influence of the tunnel jet shear layer, the extent of 2-D flow is reduced, refer to Fig. 1.b). For the ZPG case, the extent of 2-D flow is $|z| \approx 250 \mathrm{~mm}$ outside the boundary layer and only approximately $150 \mathrm{~mm}$ inside the boundary layer. The even smaller extent of 2-D flow inside the boundary layer is because, based on conservation of mass, the spread of the jet shear layer is more noticeable near the wall. For the APG case, the extent of 2-D flow is further reduced to approximately $200 \mathrm{~mm}$ and $75 \mathrm{~mm}$ outside and inside the boundary layer, respectively. The reduced extent of 2-D flow is due to the airfoil induced pressure gradient on the plate boundary layer. For example, the plate boundary layer is subjected to a favorable pressure gradient in the region around the airfoil nose. The induced low pressure, refer to Fig. 2(a), pulls the jet shear layer further into the middle, which reduces the extent of 2-D flow. Due to the smaller momentum inside the boundary layer, the near-wall region is more susceptible to this effect.

\section{B. Effect of sensor resolution and upstream boundary layer development on measured wall pressure spectra}

Fig. 4 shows the measured wall pressure power spectral density (PSD) at sensor $5(x=1165 \mathrm{~mm}$ and $z=-8 \mathrm{~mm}$, refer to Fig. 1(c)) for the different flow conditions listed in Table 1 . Pressure data recorded by sensor 4 in the mid-span $(x=1165 \mathrm{~mm}$ and $z=0 \mathrm{~mm}$ ) is not used for comparison because the measured low-frequency spectra turned out to be disturbed at sensor 4 . The problem may be related to the upstream flow disturbance caused by the three sensors placed one after the other in front of sensor 4. The spectra are computed using Welch's method [23] with a Hanning window, 8192 samples per window, and 50\% overlap. The obtained spectra have a frequency resolution of $12.2 \mathrm{~Hz}$ and the spectral levels are referenced to a $20 \mu \mathrm{Pa}$ reference pressure. The results show that the spectral magnitudes first tend to increase at low frequencies for all data sets, then decrease at medium frequencies and fall more rapidly at high 


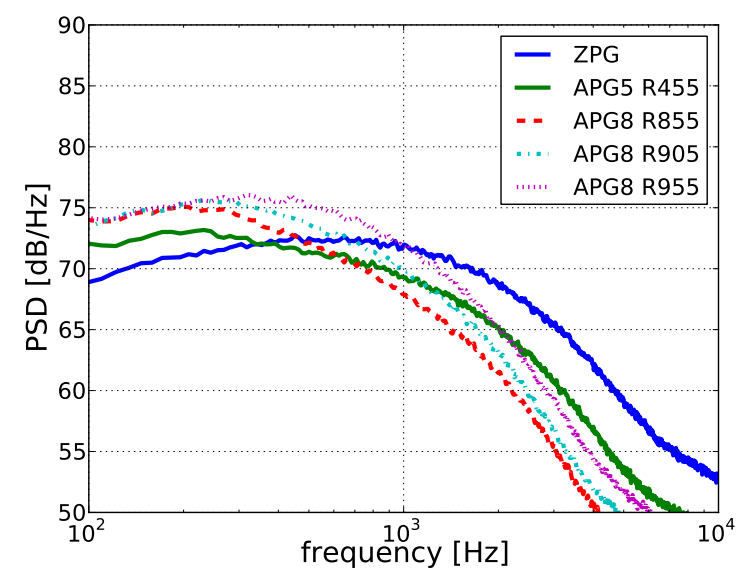

Fig. 4 Measured wall pressure spectra for the cases listed in Table 1.

frequencies. A large extent of the mid-frequency range with a constant slope is visible in the APG5 R455 and APG8 R855 cases. However, such a mid-frequency range, which should occur in the ZPG case [9], was not measured. This is due to the spectral attenuation at high frequencies, induced by signal averaging over the 'large' Kulite sensor surface.

Fig. 5illustrates the high-frequency attenuation by comparison with the ZPG spectrum measured by Hu \& Herr [7] at the same free-stream velocity $U_{\infty}=30 \mathrm{~m} / \mathrm{s}$. In their measurement, the wall pressure fluctuations were measured on a similar flat plate model using pinhole-mounted Kulites with a pinhole radius of $r=0.25 \mathrm{~mm}$, corresponding to a dimensionless radius of $r^{+}=r u_{\tau} / v=18.3$ ( $v$ is the kinematic viscosity). Gravante [24] showed that only minor attenuation was measured with a pinhole size of $r^{+}=13.1$ at very high frequencies $\omega \delta / U_{0}>43$ ( $\omega$ is the angular frequency). Thus, for the mid-frequency range $\omega \delta / U_{0}<20$ measured by Hu \& Herr, no spectral attenuation because of the finite sensor size is expected. The comparison shows that attenuation of the present result is visible from $\omega \delta / U_{0}>5$. The attenuation increases with increasing frequency and reaches $10 \mathrm{~dB}$ at $\omega \delta / U_{0}=20$, which is due to the increasing ratio of the sensor size to the incident wavelength of the wall pressure fluctuations.

The corrected spectrum with the Corcos correction [13] shows an overprediction at $\omega r / U_{c}<0.5\left(\omega \delta / U_{0}<6.4\right)$ and an underprediction at higher frequencies. Corcos calculated the sensor-size induced spectral attenuation based on the following expression

$$
\frac{\Phi_{m}(\omega)}{\Phi(\omega)}=\int \Theta(\boldsymbol{\varepsilon}) A\left(\omega \varepsilon_{x} / U_{c}\right) B\left(\omega \varepsilon_{z} / U_{c}\right) e^{-i \omega \varepsilon_{x} / U_{c}} \mathrm{~d} S(\boldsymbol{\varepsilon})
$$

where $\Phi_{m}$ and $\Phi$ are the measured and the actual wall pressure spectra. The functions $A$ and $B$ denote the streamwise and spanwise coherence functions of the wall fluctuating pressure field, respectively. $\Theta(\varepsilon)$ is the 'auto-correlation' function of the sensor overlapping area for two sensor $|\varepsilon|$ apart, where $\varepsilon=\left(\varepsilon_{x}, \varepsilon_{z}\right)$. For a circular sensor, the integral 


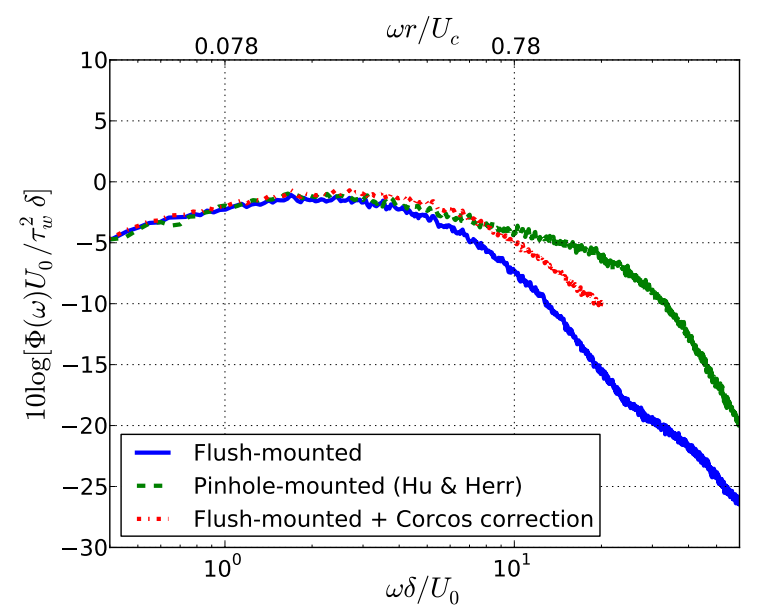

Fig. 5 Measured and corrected ZPG spectra in comparison with the result from Hu \& Herr [7].

area is defined by $S \leq 2 r$, where $r$ is the sensor radius. The attenuation values for $\omega r / U_{c}$ between 0.05 and 10 were provided by Corcos, where $U_{c}$ is the convection velocity of the wall pressure fluctuations. For application of the correction, a polynomial can be obtained by fitting the given attenuation values. However, the values of $r$ and $U_{c}$ have to be determined when applying the correction. Different selections of $r$ and $U_{c}$ could affect the level of the corrected spectra, see Fig. 6. A larger value of $r$ or a smaller value of $U_{c}$ leads to an increase in the correction level. The difference in the corrected level is larger with increasing frequency and reaches approximate $5 \mathrm{~dB}$ at $\omega \delta / U_{0}=20$ when comparing between $r=0.6$ and $1 \mathrm{~mm}$ or $U_{c}=0.4$ and $0.7 U_{0}$. Despite the different correction levels obtained by different combinations of $r$ and $U_{c}$, all corrected spectra show a steeper roll-off with increasing frequency. An extended mid-frequency range with a constant slope, which is a feature of the ZPG spectrum [9], is not present.

For the correction applied in Fig. $5, r=0.8 \mathrm{~mm}$ and $U_{c}=0.55 U_{0}$ are used. The choice of the radius of the Kulite sensor is difficult due to its specific surface configuration (18 mini-holes around a circle of $r=0.9 \mathrm{~mm}$ ). On the one hand, the radius should be smaller than $0.9 \mathrm{~mm}$ due to the small sensing area (summed area of the mini-holes, corresponding to a circle area with $r=0.4 \mathrm{~mm}$ ). On the other hand, the radius has to be larger than $0.4 \mathrm{~mm}$ because the loss in wall pressure coherence at a circle of $r=0.9 \mathrm{~mm}$ is much larger than $r=0.4 \mathrm{~mm}$. This would lead to a smaller attenuation value if $r=0.4 \mathrm{~mm}$ is applied in Eq. (11). The used radius $r=0.8 \mathrm{~mm}$ has a similar ratio of the radius to the sensor size as the one used by Herrig [25], who chose $r=0.5 \mathrm{~mm}$ for a Kulite sensor with $r=0.85 \mathrm{~mm}$. The convection velocity is determined based on the measured velocity at a sensor separation of $\Delta x=2 \mathrm{~mm}$ (the same order as the sensor size) from Hu \& Herr [7]. The convection velocity value of $U_{c}=0.55 U_{0}$ is an average value from $\omega \delta / U_{0}>5$ because attenuation is only observed in this range. Note that, this value is smaller than the often used convection velocity $U_{c}=0.8 U_{0}$ for the wall pressure fluctuations which is obtained at a large separation.

Fig. 7 a) shows the Corcos correction and the attenuation calculated based on the result shown in Fig. 5 The attenuation is only calculated up to $\omega r / U_{c}=1.5$ due to the presence of a sensor-mounting related disturbance that 


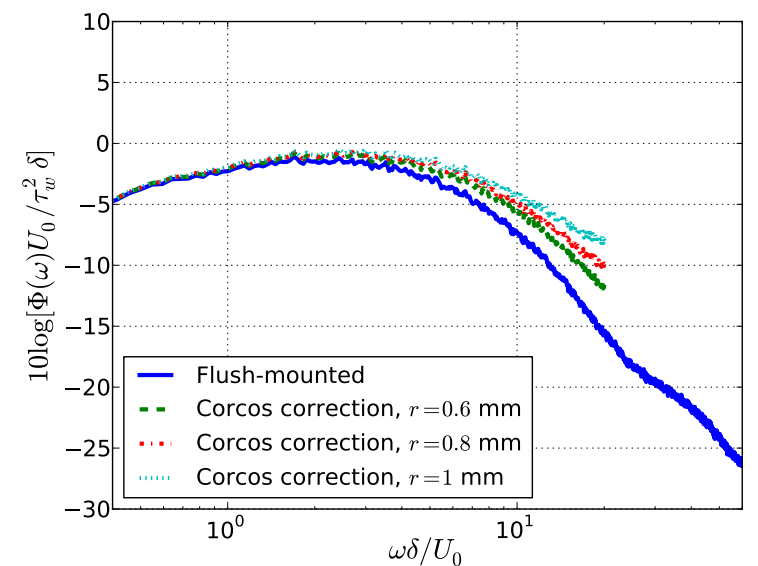

(a)

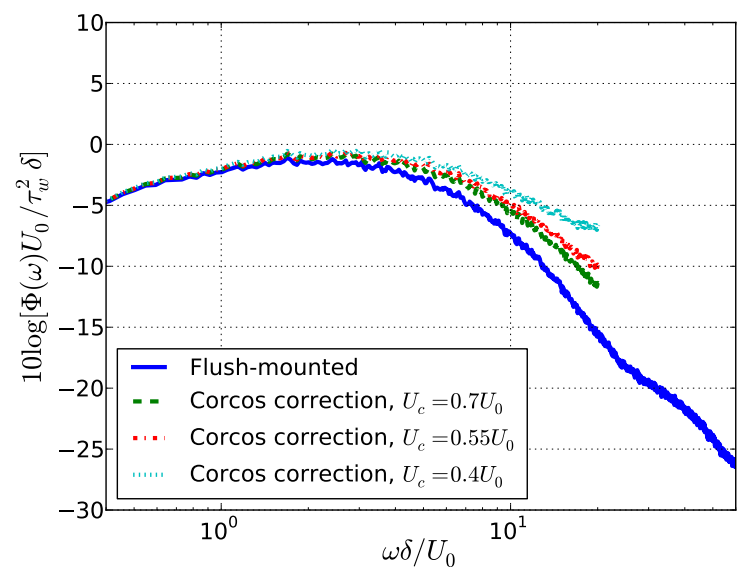

(b)

Fig. 6 Spectra corrected with different values of $r$ and $U_{c}$. (a) $U_{c}=0.55 U_{0}$; (b) $r=0.8 \mathbf{~ m m}$.

occurred at higher frequencies. The Corcos correction shows a different spectral trend from the measured attenuation, and this discrepancy is more significant at high frequencies. Two reasons may explain the insufficient accuracy of the Corcos correction. Firstly, Corcos used a rhombic-shaped coherence model $\left(A\left(\omega \varepsilon_{x} / U_{c}\right) B\left(\omega \varepsilon_{z} / U_{c}\right)\right)$ to calculate the spectral attenuation, refer to Eq. [1]. Mellen [26] and Leclercq \& Bohineust [27] studied the rhombic combination and pointed out its insufficient accuracy for the prediction of the off-axis coherence. Secondly, non-uniform sensitivity across a sensor surface, which is most of the time the case in practice, is not involved in Eq. (1). The measurement of Gilchrist \& Strawderman [14] showed that the sensitivity of two pressure sensors is larger in the middle and drops near the edge. Farabee et al. [15] compared the measured wavenumber response of a circular sensor with the theoretical function for the response under the assumption of uniform sensitivity over its surface. The measured response showed a slightly larger value $(<0.5 \mathrm{~dB})$ of the acceptance at low wavenumbers and a smaller value at high wavenumbers $(5 \mathrm{~dB}$ at $\omega U_{c} / r=2$ ) in comparison with the theoretical function. It is interesting to note that this discrepancy between the measured and theoretical results of the sensor response is similar to the discrepancy shown in Fig. 77a) between the measured attenuation and the Corcos correction. Due to the specific surface configuration of the applied Kulite sensor, it is even harder to estimate the sensitivity distribution. Nevertheless, a non-uniform distribution of the sensitivity would be expected.

The obtained attenuation shows a slow drop at low frequencies and a steep roll-off at high frequencies. The roll-off tends towards a constant slope with increasing frequency. This kind of spectral shape can be expressed with a logarithm function as

$$
10 \log \frac{\Phi_{m}(\omega)}{\Phi(\omega)}=10 \log \frac{a}{a+\left(\omega r / U_{c}\right)^{b}}
$$

Using curve fitting, $a=0.427$ and $b=2.85$ are determined and the resulting curve is plotted in Fig. 7 7 a). It is 


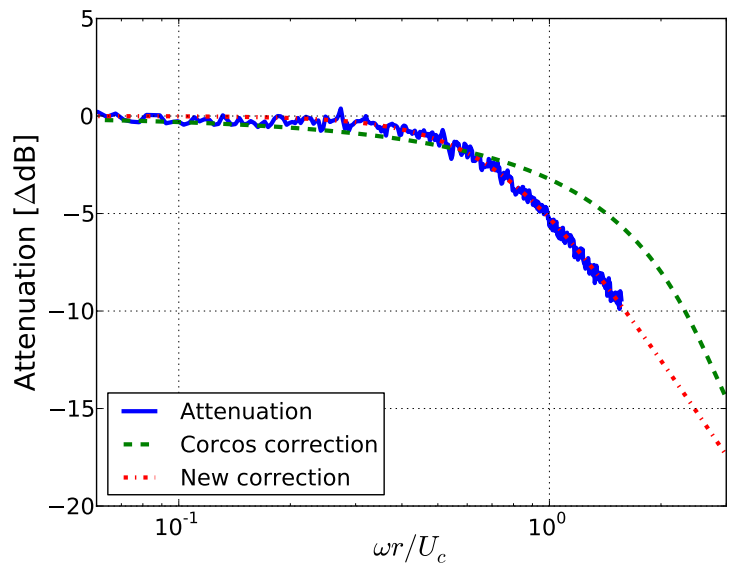

(a)

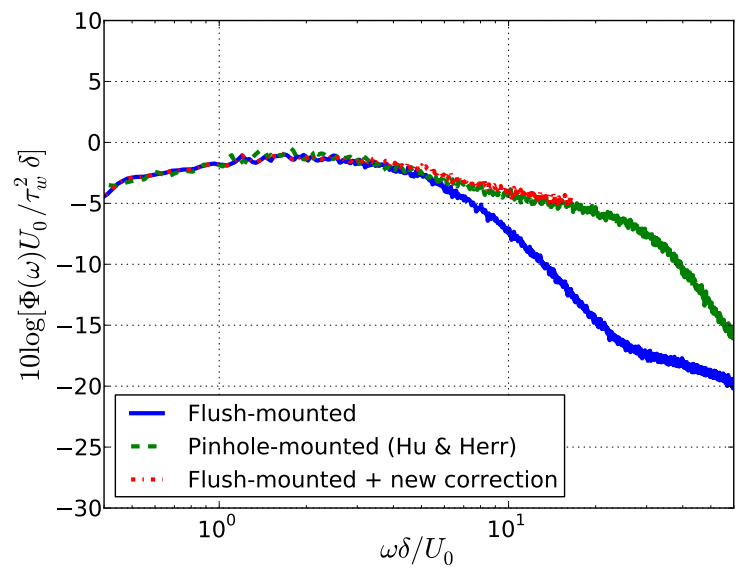

(b)

Fig. 7 (a) Measured spectral attenuation in comparison with the Corcos correction at $U_{\infty}=30 \mathrm{~m} / \mathbf{s}$; (b) measured ZPG spectrum and according to Eq. (2) corrected spectrum in comparison with the result from Hu \& Herr [7] at $U_{\infty}=40 \mathrm{~m} / \mathrm{s}$.

worth mentioning that the measurement uncertainties could affect the accuracy of the obtained correction spectrum. Uncertainty in flow parameters is mainly caused by the initial positions $(<0.2 \mathrm{~mm})$ and the accuracy $(1 \%)$ of the hot-wire anemometer. The dominant source of uncertainty for the wall pressure spectral measurements is the absolute value of the sensor sensitivity $(<0.5 \mathrm{~dB})$. An uncertainty of about $5 \%$ in the frequency scaling and of about $1 \mathrm{~dB}$ in the magnitude scaling is suggested. Hence, a major impact on the spectral shape and magnitude of the obtained correction spectrum due to the measurement uncertainties is not expected. The acquired correction is also applied to the measured ZPG spectrum at a higher velocity of $U_{\infty}=40 \mathrm{~m} / \mathrm{s}$ and a perfect match between the corrected spectrum and the measured spectrum from Hu \& Herr is shown in Fig. 7(b).

Fig. 8 shows the measured and corrected spectra for the APG8 cases in comparison with the predicted spectra from different models. For the correction, an even smaller value of $U_{c}=0.37 U_{0}$ is used, because the convection velocity in an APG flow is smaller than in a ZPG flow [7, 11, 28]. The selected value of $U_{c}$ is calculated with the same principle as for the ZPG case and based on the result from Hu \& Herr [7] for the APG $10^{\circ}$ case, which has similar boundary layer conditions as in the present APG8 cases. The corrected spectra with the new correction show a large extent of the mid-frequency range with a constant slope. The presence of this mid-frequency range in an APG flow agrees with the results from Catlett et al. [5], Hu \& Herr [7] and Suryadi \& Herr [29], all of whom measured the wall pressure using sensor configurations with pinholes. Note that, instead of a pinhole-mounted Kulite sensor used in the latter two contributions, a flush-mounted pressure sensor with a pinhole of $r=0.43 \mathrm{~mm}$ on its screen was used by Catlett et al. Because they measured the wall pressure beneath a thick boundary layer flow $(\delta>6 \mathrm{~cm})$, the sensor-size induced attenuation occurs at frequencies sufficiently beyond the mid-frequency range of interest. In contrast to the 


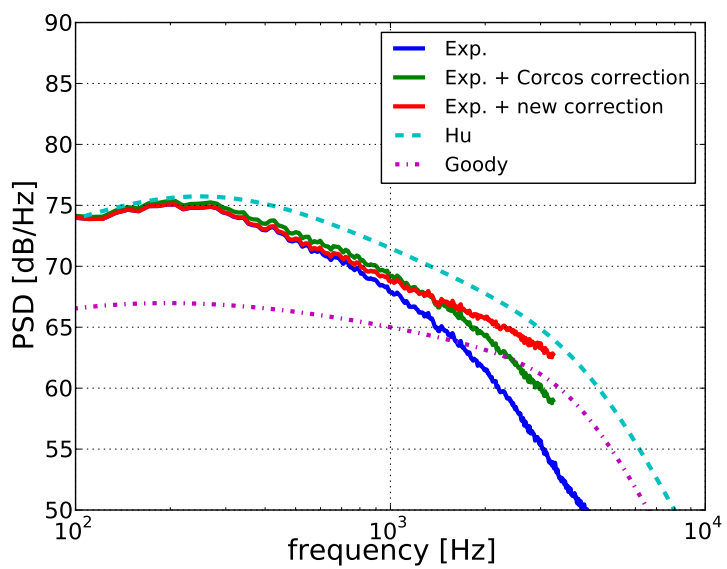

(a)

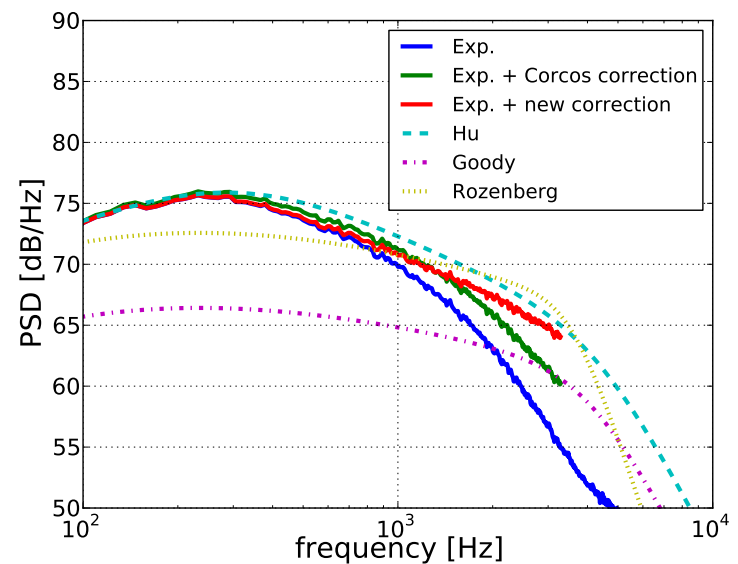

(b)

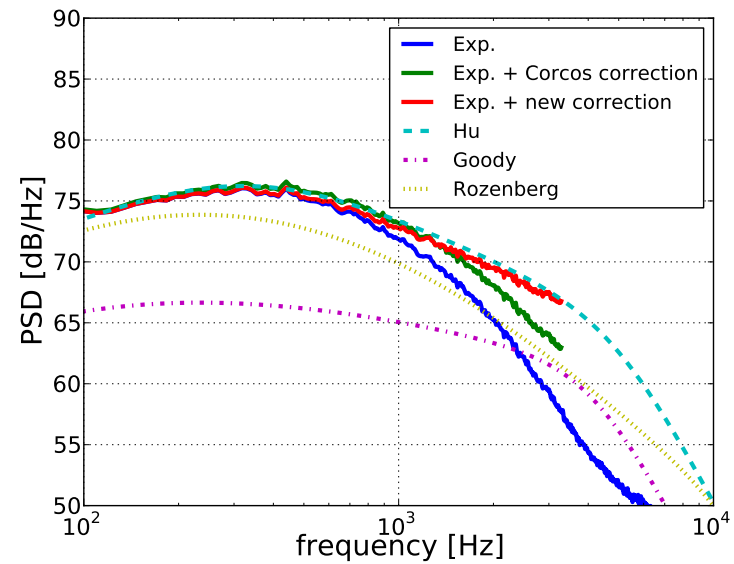

(c)

Fig. 8 Measured, corrected and predicted spectra. (a) APG8 R855; (b) APG8 R905; (c) APG8 R955.

spectra obtained by Eq. (2), those spectra corrected with the Corcos correction display a steeper roll-off with increasing frequency, which leads to a level approximately $4 \mathrm{~dB}$ smaller than the results obtained from the new correction at $3 \mathrm{kHz}$.

Errors can be introduced when applying the correction function Eq. (2) to the APG cases due to the difference in wall pressure coherence between APG and ZPG flows. An estimation of the attenuation for the APG $10^{\circ}$ case from Hu \& Herr [7] with the corresponding wall pressure coherence is made according to Eq. (1), which is then compared to the Corcos correction, see Fig. 9. The result shows that the obtained attenuation is smaller than the value of the Corcos correction which was developed based on the coherence of a ZPG wall pressure. At low frequencies the difference between both attenuation spectra is small but becomes larger at high frequencies. For the frequency range $\omega r / U_{c}<1.5$ where the correction is applied in this paper, a minor difference of $0.5 \mathrm{~dB}$ is found which has no significant influence on the corrected spectra for the APG8 cases.

The prediction results in Fig. 8] show that Goody's model [9] lacks accuracy for the APG flows. This is expected 


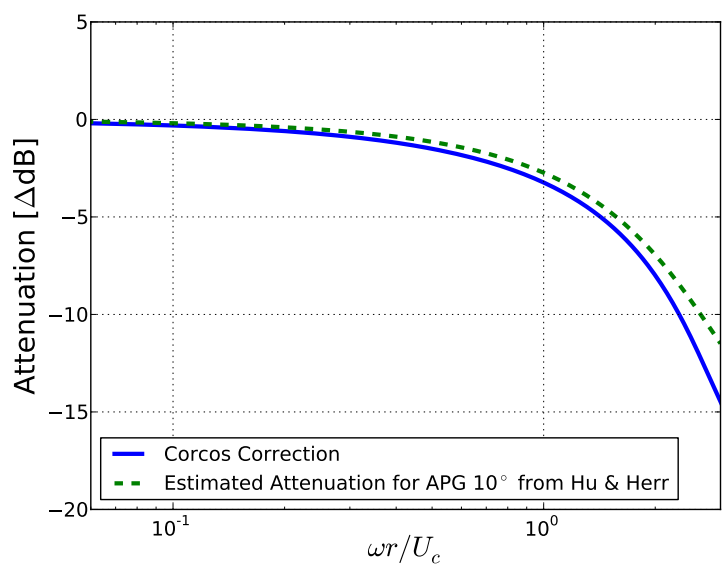

Fig. 9 Estimation of the attenuation for the APG $10^{\circ}$ case from Hu \& Herr [7].

because the model was developed for ZPG flows and cannot predict the influence of pressure gradients on the wall pressure spectrum. Rozenberg's model [8] and Hu's model [12] were developed to capture the spectral change in an APG flow. Rozenberg used Clauser's parameter as the driving parameter to predict the effect of APGs on the wall pressure spectrum, whereas Hu used the boundary layer shape factor. For example, a larger value of Clauser's parameter in Rozenberg's model will predict a steeper mid-frequency spectral slope. The same spectral trend will be given for a larger value of the boundary layer shape factor in Hu's model.

A good prediction accuracy for all measured spectra is given by Hu's model. Especially good agreement can be found for the prediction of the mid-frequency spectral slope when compared to the corrected spectra with the new correction. In contrast, Rozenberg's model achieves a good prediction only for the APG8 R955 case in which $\beta_{\delta^{*}}=2.97$. For the APG8 R905 case, the predicted spectrum from Rozenberg's model shows the same mid-frequency slope as Goody's model. This is because Rozenberg's model uses Clauser's parameter as the driving parameter to predict the mid-frequency slope which in this case is nearly zero $\left(\beta_{\delta^{*}}=0.17\right)$ due to the nearly zero local pressure gradient. Rozenberg's model fails to predict the APG8 R855 case because the model cannot deal with Clauser's parameter $\beta_{\theta}<-0.5$ due to its one parameter defined as $0.8 \cdot\left(\beta_{\theta}+0.5\right)^{3 / 4}$. In our case, $\beta_{\theta}=-1.08$. This result indicates that Clauser's parameter, which is strongly affected by the local pressure gradient, is an insufficient indicator to predict the APG effect on the wall pressure spectrum in a non-equilibrium flow. Hence, the boundary layer shape factor would be a more proper indicator because it also reflects the upstream flow history.

\section{Effect of non-flush mounting and upstream disturbance on measured wall pressure spectra}

In practical applications an imperfect flush-mounting of the sensor or upstream flow disturbances could affect the accuracy of the measured wall pressure spectrum. Such effects were studied using sensors 5-9 at $x=1165 \mathrm{~mm}$ and $-8 \mathrm{~mm}<z<-40 \mathrm{~mm}$. A sensor offset with respect to the wall was intentionally introduced from $\Delta y=-0.2 \mathrm{~mm}$ to 


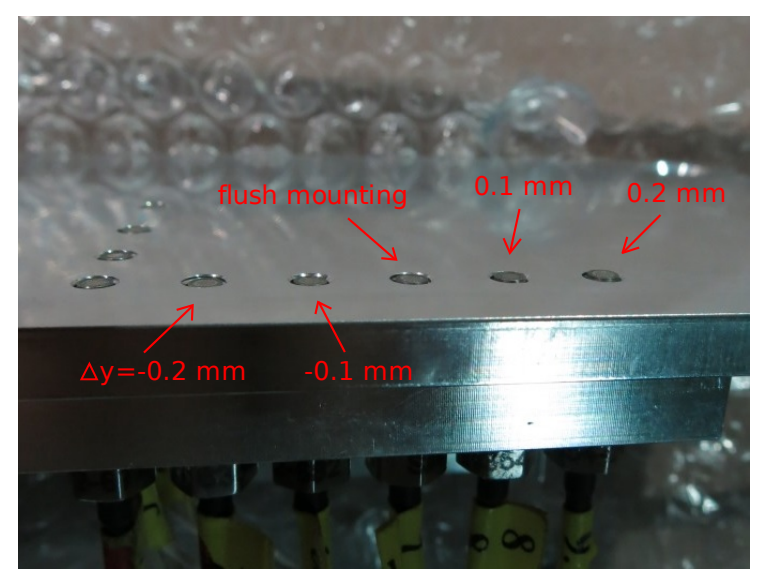

(a)

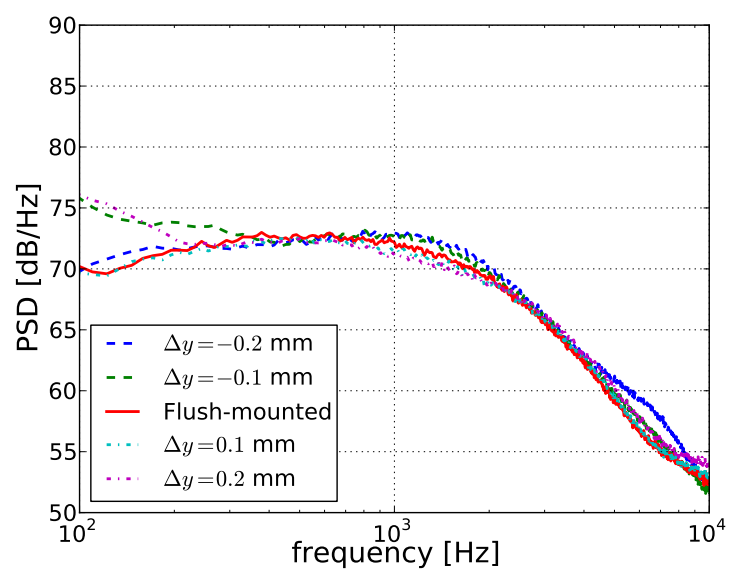

(c)

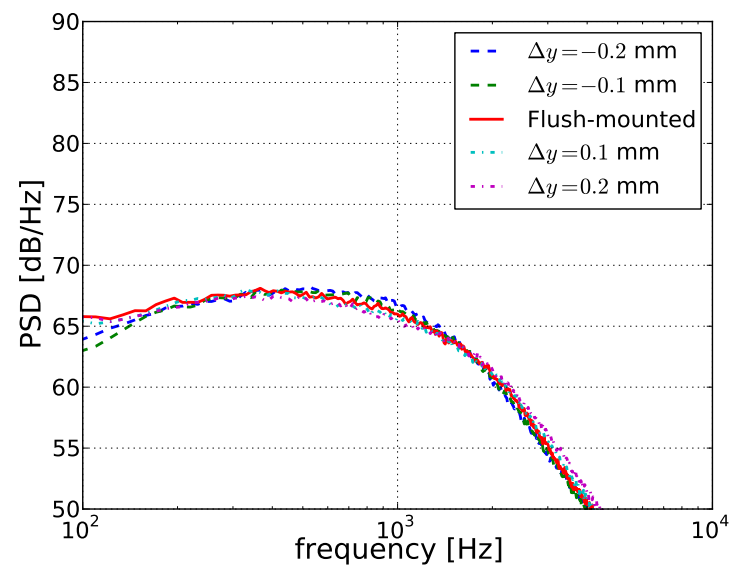

(b)

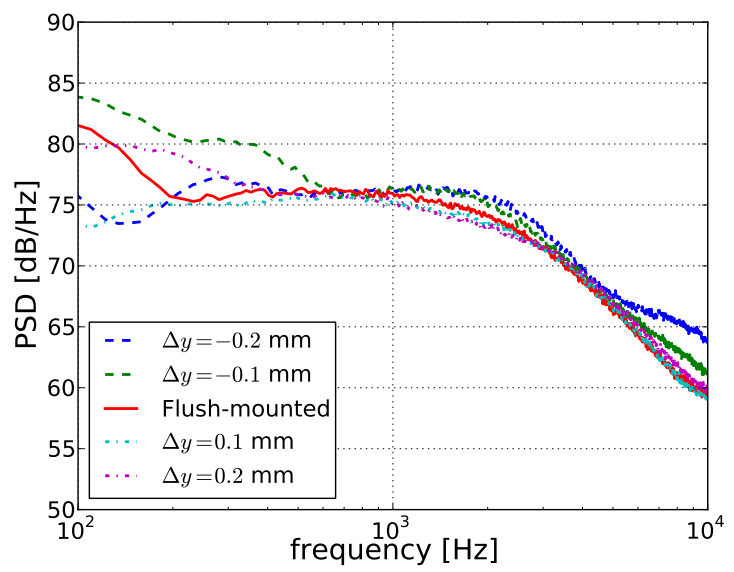

(d)

Fig. 10 (a) Photo of the non-flush mounting configuration; (b-d) measured spectra for the ZPG case; (b) $U_{\infty}=20 \mathbf{m} / \mathbf{s} ;$ (c) $U_{\infty}=30 \mathrm{~m} / \mathbf{s} ;$ (d) $U_{\infty}=40 \mathrm{~m} / \mathbf{s}$.

$\Delta y=0.2 \mathrm{~mm}$ with $0.1 \mathrm{~mm}$ increment, see Fig. 10.a). The accuracy of the mounting non-flushness was guaranteed through a thread turning insert. A $0.3 \mathrm{~mm}-0.4 \mathrm{~mm}$ gap between the sensor and the plate surface was present due to the manufacturing of the thread hole which can be also categorized as an imperfect mounting, see Fig. 11

Figs. 10 (b-d) show the measured wall pressure spectra for the ZPG case at the nozzle free-stream velocities of $U_{\infty}=20,30,40 \mathrm{~m} / \mathrm{s}$. Three main features can be observed for the sensor offset induced spectral distortion. Firstly, the distortion is larger at a higher velocity. Secondly, the distortion is larger at low frequencies. Thirdly, a negative sensor offset increases the spectral level at medium frequencies, whereas a positive offset decreases the level. These findings are consistent with the results from Efimtsov et al. [30] which were obtained by flight and wind tunnel measurements at Mach numbers ranging from 0.5 to 2.5. Due to the presence of a small gap between the sensor and the plate surface in the present test, no systematic trend of the spectral distortion at low frequencies can be found between the negative and 


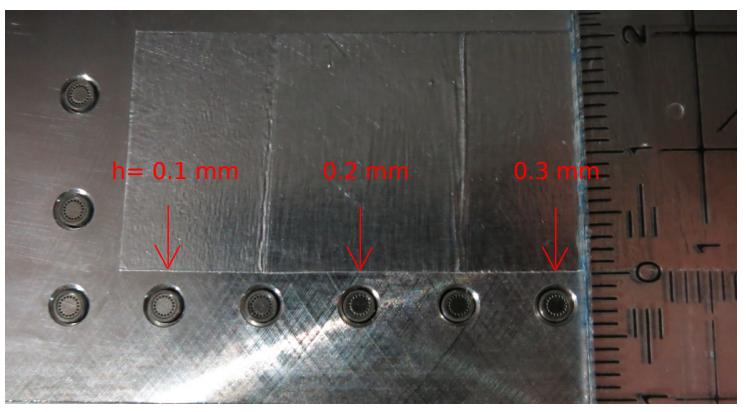

(a)

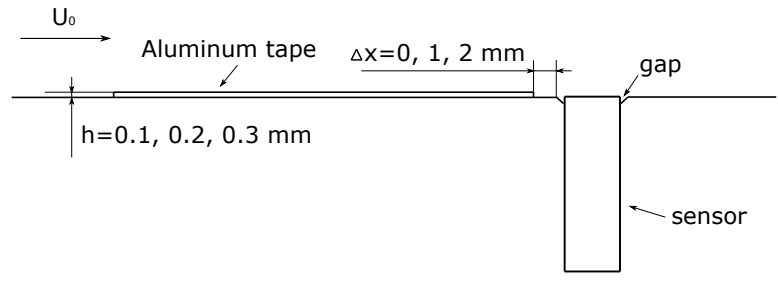

(b)

Fig. 11 Test configurations for the cases with aluminum tapes. (a) photo; (b) schematic.

positive offset configurations. The low-frequency spectra are susceptible to the gap induced disturbance. For example, a spectral distortion of more than $5 \mathrm{~dB}$ at $100 \mathrm{~Hz}$ was measured by the flush-mounted sensor 7 for $U_{\infty}=40 \mathrm{~m} / \mathrm{s}$, refer to Fig. 10.d), whereas the low-frequency distortion is much smaller at the sensor 5 shown in Fig. 12 (c) for the case without disturbance. Furthermore, the level of the spectral distortion could be affected by the finite sensor size, because the spectral attenuation for the short scale disturbance is different from the boundary layer induced wall pressure fluctuations. Hence, the spectral correction is not applied to the results in this section.

The effect of upstream flow disturbances was studied by placing aluminum tapes in front of the sensors 5-9 with a distance of $\Delta x=0,1,2 \mathrm{~mm}$, illustrated in Fig. 11. The aluminum tape has a thickness of $0.1 \mathrm{~mm}$. Through single-, double- and triple-layer aluminum tapes, heights of $0.1 \mathrm{~mm}, 0.2 \mathrm{~mm}$ and $0.3 \mathrm{~mm}$ steps in front of sensors 5,7 and 9 were placed, respectively. The length of the aluminum tape in the streamwise direction was $2 \mathrm{~cm}$. This step configuration gives a smallest step length-to-height ratio of $x / h=67$ and a largest step height to boundary layer thickness ratio of $h / \delta=1.6 \%$. Ji \& Wang [31] calculated a forward-facing step induced flow disturbance at a comparable Reynolds number flow for small steps with $h / \delta=0.83 \%$ and $3.3 \%$. The results showed that the flow over these small forward-facing steps recovers by a distance of $x / h \simeq 50$. This indicates that in our test the aluminum tape induced disturbance can be 'simply' treated as a backward-facing step induced disturbance. However, due to interaction with the gap between the sensor and the plate surface, more complex disturbance may be generated.

Fig. 12 shows the results for the ZPG case with the aluminum tape at $\Delta x=1 \mathrm{~mm}$. The spectral distortion is visible for all disturbed flow cases. The distortion is larger at a higher velocity which agrees with the sensor offset test. A broadband increase over the whole frequency range is found for the cases with $h=0.2,0.3 \mathrm{~mm}$, whereas an increase only occurs at low frequencies for the case with $h=0.1 \mathrm{~mm}$. Furthermore, the increased level for the cases with $h=$ $0.2,0.3 \mathrm{~mm}$ is much larger. For example, the spectral increase reaches $15 \mathrm{~dB}$ at $100 \mathrm{~Hz}$ for the case with $h=0.2 \mathrm{~mm}$ and $U_{\infty}=40 \mathrm{~m} / \mathrm{s}$.

Fig. 13 shows the results for the cases with the aluminum tape at $\Delta x=0,2 \mathrm{~mm}$. The same results as the case with $\Delta x=1 \mathrm{~mm}$, the spectral distortion is larger for a thicker step. Similar features of the distorted spectra can be observed 


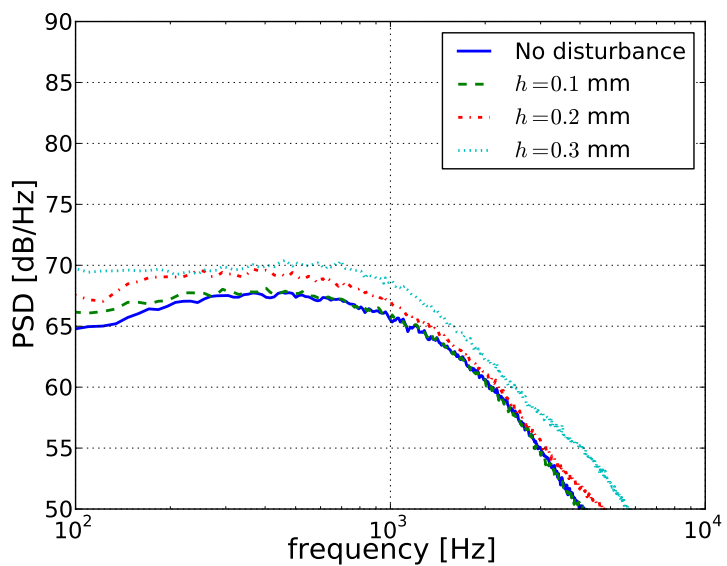

(a)

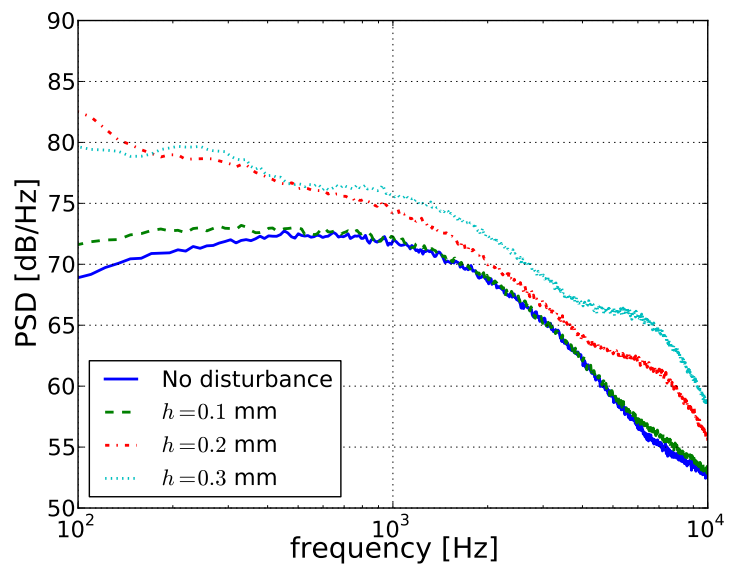

(b)

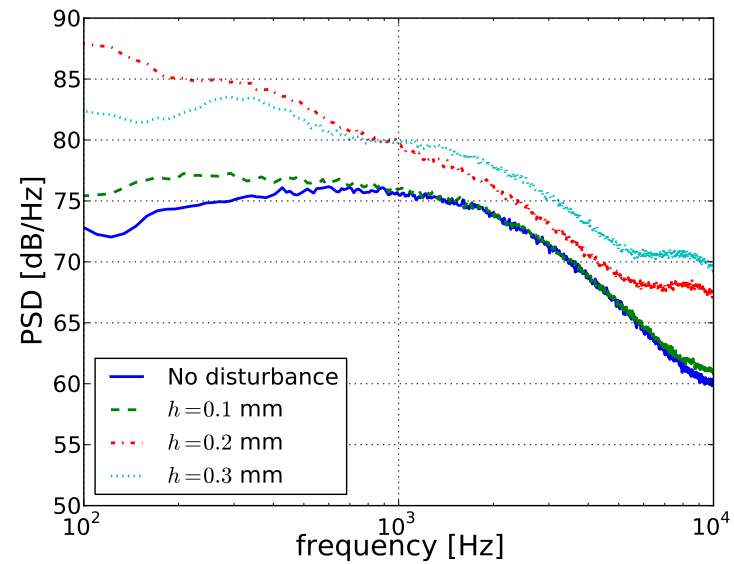

(c)

Fig. 12 Measured spectra for the ZPG case with the aluminum tape at $\Delta x=1 \mathbf{m m}$. (a) $U_{\infty}=20 \mathrm{~m} / \mathbf{s}$; (b) $U_{\infty}=30 \mathbf{m} / \mathbf{s}$; (c) $U_{\infty}=40 \mathbf{m} / \mathbf{s}$.

between the cases with $\Delta x=1 \mathrm{~mm}$ and $2 \mathrm{~mm}$, refer to Figs. $[12(\mathrm{~b})-13 \mathrm{~b}$ ). The spectral increase level is smaller for $\Delta x=2 \mathrm{~mm}$ due to the larger distance between the step and the sensor. For the case with $\Delta x=0 \mathrm{~mm}$, three distinct distortion patterns are visible at low, medium and high frequencies, which are comparable to the case with a negative sensor offset, see Fig. 10. This distortion feature is probably related to the presence of the gap, with which the separation bubble from the backward step interacts.

The test with the aluminum tape was also conducted for the other flow conditions listed in Table 1 in the same manner as for the ZPG case. Fig. 14 shows the results for the cases of APG5 R455 and APG8 R955 at $U_{\infty}=30 \mathrm{~m} / \mathrm{s}$. Unlike the ZPG case in which the spectra were significantly distorted, almost no spectral distortion was measured for the APG8 R955 case and for the other APG8 cases (not shown). The high resistance to the spectral distortion in the APG8 cases is probably attributed to the larger value of the boundary layer thickness $\delta$ and the shape factor $H$, and the 


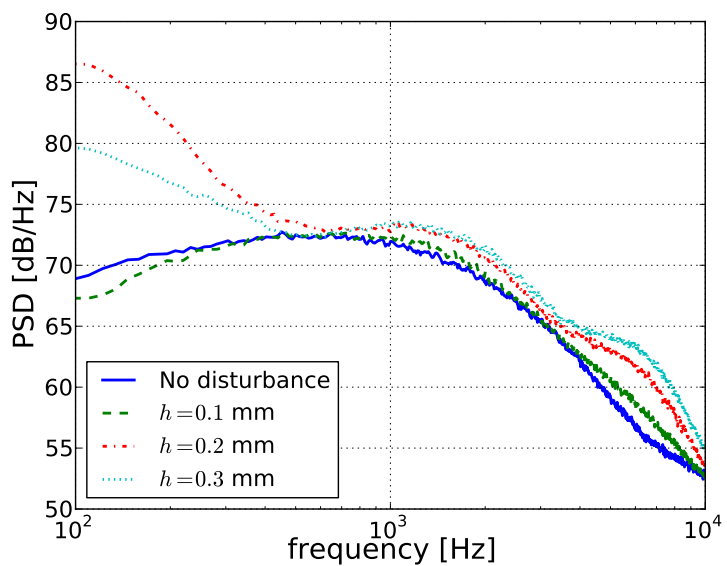

(a)

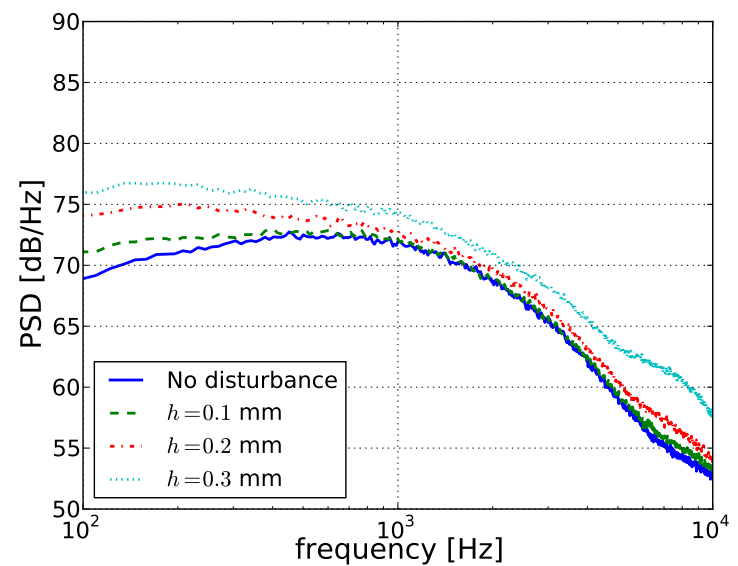

(b)

Fig. 13 Measured spectra for the ZPG case at $U_{\infty}=30 \mathrm{~m} / \mathbf{s}$. (a) $\Delta x=0 \mathbf{m m}$; (b) $\Delta x=2 \mathbf{m m}$.

smaller value of the friction velocity $u_{\tau}$. The level of the spectral distortion measured in the APG5 R455 case is larger than the APG8 R955 case, but smaller than the ZPG case. For the APG5 R455 case, the flow has the largest value of $\delta$ but the values of $H$ and $u_{\tau}$ fall between the cases of ZPG and APG8 R955. This indicates that the influence of $H$ and $u_{\tau}$ on the resistance to the spectral distortion is bigger than $\delta$ for the test cases. Furthermore, the larger spectral distortion was measured in the ZPG case at a higher flow velocity, refer to Fig. 12. This can be explained by the fact that the flow with a higher velocity has a larger value of $u_{\tau}$, which could increase the influence of flow disturbances on the wall pressure spectra.

\section{Conclusion}

Wall pressure fluctuations were measured by flush-mounted Kulite sensors on a flat plate model. Streamwise pressure gradients on the plate were generated by a NACA 0012 airfoil placed above the plate. Different flow characteristics and local pressure gradients at the measurement position were induced by adjusting the angle of attack and the streamwise position of the airfoil. The mean velocity parameters were obtained by a hot-wire anemometer.

The measured wall pressure spectra show spectral attenuation at high frequencies due to the finite sensor size. The Corcos correction is applied to compensate for the attenuation in a zero pressure gradient (ZPG) flow. The result is compared to the measured wall pressure spectrum by using a pinhole-mounted Kulite sensor in a similar experimental setup from Hu \& Herr [7]. The comparison indicates that the Corcos correction overpredicts the spectrum at $\omega r / U_{c}<0.5$ and underpredicts at $\omega r / U_{c}>0.5$. A new correction is proposed based on the measured results for the ZPG flows and validated up to $\omega r / U_{c}=1.5$. Furthermore, the new correction is applied to the measured spectra in adverse pressure gradients (APG). The corrected spectra show an extended mid-frequency range with a constant slope. The presence of this mid-frequency range in APG flows agrees with the experimental results [5, 7, 29], which were 


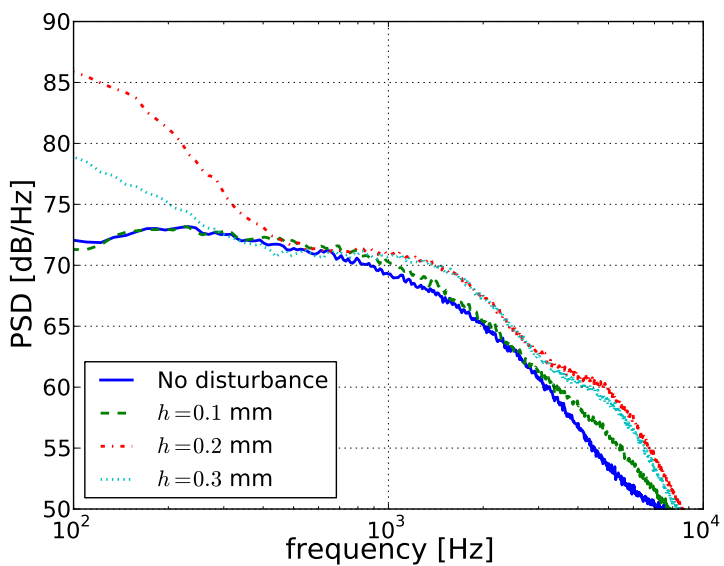

(a)

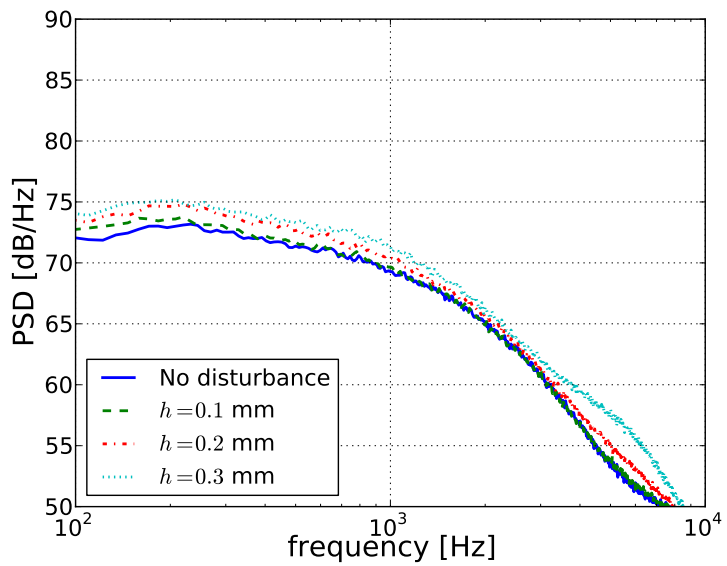

(c)

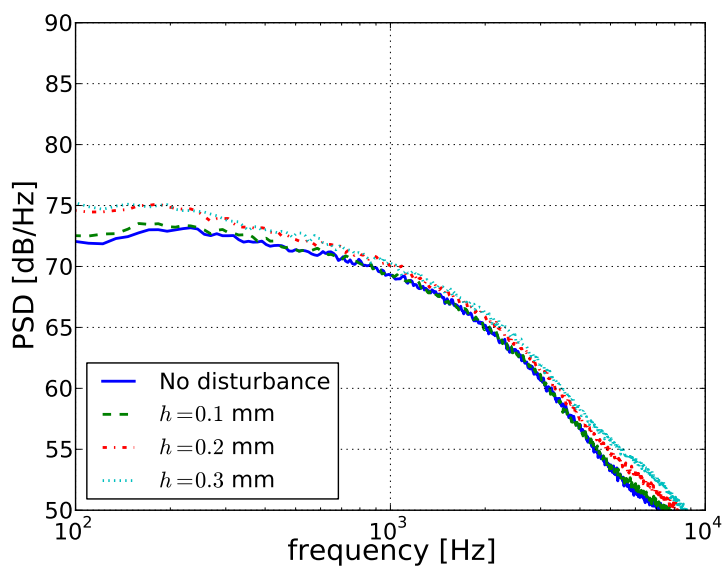

(e)

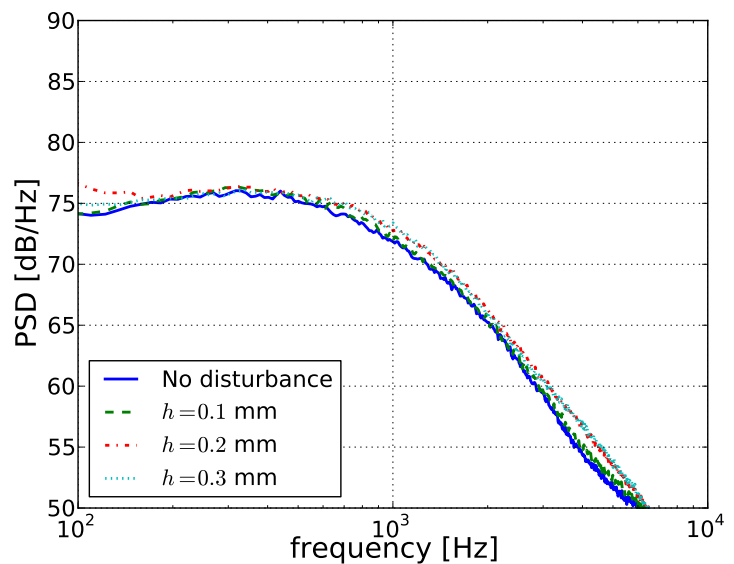

(b)

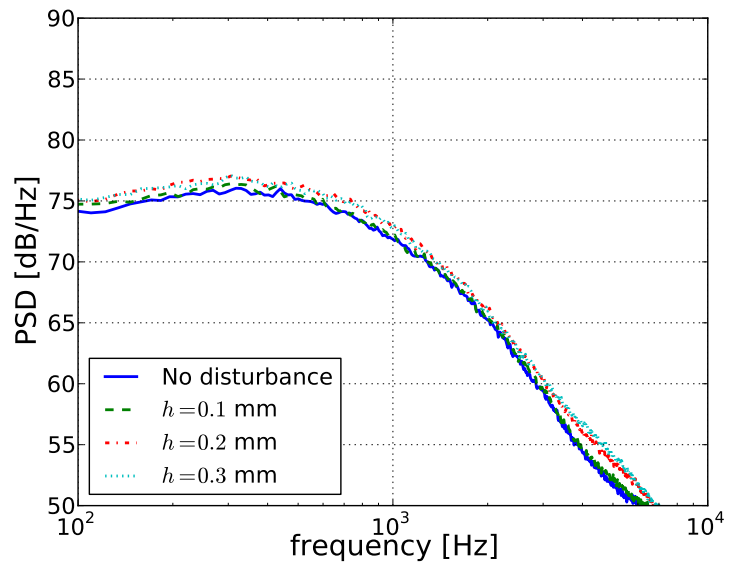

(d)

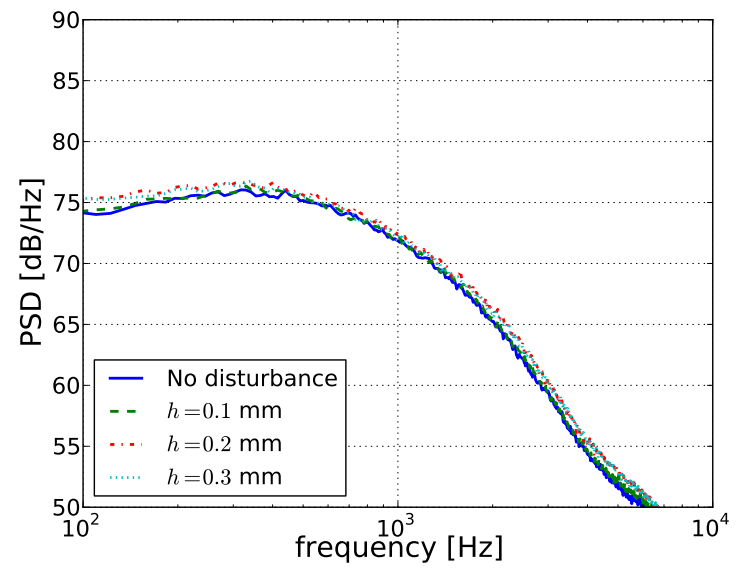

(f)

Fig. 14 Measured spectra at $U_{\infty}=30 \mathrm{~m} / \mathrm{s}$. (a, b) $\Delta x=0 \mathrm{~mm}$, (a) APG5 R455; (b) APG8 R955. (c, d) $\Delta x=1 \mathrm{~mm}$, (c) APG5 R455; (d) APG8 R955. (e, f) $\Delta x=2$ mm, (e) APG5 R455; (f) APG8 R955. 
measured using sensors with sufficiently small sensing areas. Furthermore, the obtained value of the slope via the new correction is consistent with the prediction of Hu's model [12].

The measured and corrected APG spectra indicate that Clauser's parameter, which is strongly affected by the local pressure gradient, is an insufficient indicator to predict the APG effect on the wall pressure spectra in a non-equilibrium flow. In contrast, the boundary layer shape factor which is mainly determined by the flow development history would be a more appropriate parameter for the prediction of the APG spectra.

Furthermore, the spectral distortion of the wall pressure due to sensor mounting imperfections and flow disturbances induced by small steps in front of the sensor is studied. The results show that for a ZPG flow a disturbance induced by a sensor offset of $\Delta y / \delta=0.5 \%$ with respect to the wall or a step height of $h / \delta=0.5 \%$ can affect the wall pressure spectrum. The induced spectral distortion is larger at a higher flow velocity and also when the sensor offset or the step height increases. The results from the tested flow configurations indicate that the shape factor and the friction velocity are the key parameters for the resistance to the spectral distortion induced by flow disturbances. An increase of the shape factor or a decrease of the friction velocity can reduce the influence of flow disturbances.

\section{Acknowledgments}

This work was conducted in the framework of a cooperation between Daimler AG and DLR.

\section{References}

[1] Willmarth, W. W., and Wooldridge, C. E., "Measurements of the fluctuating pressure at the wall beneath a thick turbulent boundary layer," J. Fluid Mech., Vol. 14, 1962, pp. 187-210. doi:10.1017/S0022112062001160.

[2] Bull, M. K., "Wall pressure fluctuations associated with subsonic turbulent boundary layer flow," J. Fluid Mech., Vol. 28, 1967, pp. 719-754. doi:10.1017/S0022112067002411.

[3] Blake, W. K., "Turbulent boundary layer wall pressure fluctuations on smooth or rough walls," J. Fluid Mech., Vol. 44(4), 1970, pp. 637-660. doi:10.1017/S0022112070002069.

[4] Farabee, T. M., and Casarella, M. J., "Spectral features of wall pressure fluctuations beneath turbulent boundary layers," Phys. Fluids, Vol. A3(10), 1991, pp. 2410-2420. doi:10.1063/1.858179.

[5] Catlett, M. R., Forest, J. B., Anderson, J. M., and Stewart, D. O., "Empirical spectral model of surface pressure fluctuations beneath adverse pressure gradients," AIAA Paper 2014-2910, 2014. doi:10.2514/6.2014-2910.

[6] Salze, E., Bailly, C., Marsden, O., Jondeau, E., and Juvé, D., “An experimental characterisation of wall pressure wavevectorfrequency spectra in the presence of pressure gradients," AIAA Paper 2014-2909, 2014. doi:10.2514/6.2014-2909.

[7] Hu, N., and Herr, M., "Characteristics of wall pressure fluctuations for a flat plate turbulent boundary layer with pressure gradients," AIAA Paper 2016-2749, 2016. doi:10.2514/6.2016-2749. 
[8] Rozenberg, Y., Robert, G., and Moreau, S., "Wall-pressure spectral model including the adverse pressure gradient effects," AIAA Journal, Vol. 50(10), 2012, pp. 2168-2179. doi:10.2514/1.J051500.

[9] Goody, M. C., "Empirical Spectral Model of Surface Pressure Fluctuations," AIAA Journal, Vol. 42(9), 2004 , pp. $1788-1794$. doi:10.2514/1.9433

[10] Kamruzzaman, M., Bekiropoulos, D., Lutz, T., and Würz, W., “A semi-empirical surface pressure spectrum model for airfoil trailing-edge noise prediction,” International journal of aeroacoustics, Vol. 14, 2015. doi:10.1260/1475-472X.14.5-6.833.

[11] Catlett, M. R., Anderson, J. M., Forest, J. B., and Stewart, D. O., "Empirical modeling of pressure spectra in adverse pressure gradient turbulent boundary layers," AIAA Journal, Vol. 54(2), 2016. doi:10.2514/1.J054375.

[12] Hu, N., "Empirical model of wall pressure spectral in adverse pressure gradients," AIAA Journal, Vol. 56 (9), 2018, pp. 3491-3506. doi:10.2514/1.J056666.

[13] Corcos, G. M., "Resolution of Pressure in turbulence,” J. Acoust. Soc. Am., Vol. 35, 1964, pp. 192-199. doi:10.1121/1.1918431.

[14] Gilchrist, R. B., and Strawderman, W. A., "Experimental hydrophone-size correction factor for boundary layer pressure fluctuations," J. Acoust. Soc. Am., Vol. 38, 1965, pp. 298-302. doi:10.1121/1.1909663.

[15] Farabee, T. M., Geib, F. E., and Taylor, J. W., "Measurement of boundary layer press ure fields with an array of pressure transducers in a subsonic flow," Proc. Int. Congr. Instrum, Aerosp. Facil., 6th, Ottawa, Can., 1975, pp. 311-319.

[16] Narasimha, R., and Prasad, S. N., "Leading edge shape for flat plate boundary layer studies," Experiments in Fluids, Vol. 17(5), 1994, pp. 358-360.

[17] Mosallem, M. M., "Numerical and experimental investigation of beveled trailing edge flow fields," Journal of Hydrodynamics, Vol. 20(3), 2008, pp. 273-279. doi:10.1016/S1001-6058(08)60057-8.

[18] Erbig, L., Hu, N., and Lardeau, S., "Experimental and Numerical Study of Passive Gap Noise," AIAA Paper 2018-3595, 2018. doi:10.2514/6.2018-3595.

[19] Reichardt, H., "Vollständige Darstellung der turbulenten Geschwindigkeitsverteilung in glatten Leitungen,” Z. Angew. Math. Mech., Vol. 31(7), 1951, pp. 208-219. doi:10.1002/zamm.19510310704.

[20] Clauser, F. H., “Turbulent boundary layers in adverse pressure gradients,” Journal of the Aeronautical Sciences, Vol. 21(2), 1954, pp. 91-108. doi:10.2514/8.2938.

[21] Mellor, G. L., and Gibson, D. M., “Equilibrium turbulent boundary layers,” J. Fluid Mech., Vol. 24(2), 1966, pp. $225-253$. doi:10.1017/S0022112066000612.

[22] Herring, H. J., and Norbury, J. F., "Some experiments on equilibrium turbulent boundary layers in favorable pressure gradients," J. Fluid Mech., Vol. 27(3), 1967, pp. 541-549. doi:10.1017/S0022112067000527. 
[23] Welch, P. D., "The use of fast Fourier transform for the estimation of power spectra: A method based on time averaging over short, modified periodograms," IEEE Transactions on Audio and Electroacoustics, Vol. 15 (2), 1967, pp. 70-73. doi:10.1109/TAU.1967.1161901.

[24] Gravante, S. P., Naguib, A. M., Wark, C. E., and Nagib, H. M., "Characterization of the pressure fluctuations under a fully developed turbulent boundary layer," AIAA Journal, Vol. 36(10), 1998, pp. 1808-1816. doi:10.2514/2.296.

[25] Herrig, A., "Validation and application of a hot-wire based method for trailing edge noise measurements on airfoils," Ph.D. thesis, University of Stuttgart, 2012.

[26] Mellen, R. H., “On modeling convective turbulence,” J. Acoust. Soc. Am., Vol. 88(6), 1990, pp. 2891-2893. doi:10.1121/1.399695.

[27] Leclercq, D. J. J., and Bohineust, X., "Investigation and modelling of the wall pressure field beneath a turbulent boundary layer at low and medium frequencies," J. Sound Vib., Vol. 257(3), 2002, pp. 477-501. doi:10.1006/jsvi.2002.5049.

[28] Schloemer, H. H., "Effects of pressure gradients on turbulent-boundary-Layer wall-pressure fluctuations," J. Acoust. Soc. Am., Vol. 42(1), 1967, pp. 93-113. doi:10.1121/1.1910581.

[29] Suryadi, A., and Herr, M., "Wall pressure spectra on a DU96-W-180 profile from low to pre-stall angles of attack," AIAA Paper 2015-2688, 2015. doi:10.2514/6.2015-2688.

[30] Efimtsov, B. M., Golubev, A. Y., Kuznetsov, V. B., Rizzi, S. A., Andersson, A. O., Rackl, R. G., and Andrianov, E. V., "Effect of transducer flushness on measured surface pressure fluctuations in flight," AIAA Paper 2005-800, 2005. doi:10.2514/6.2005-800.

[31] Ji, M., and Wang, M., "Surface pressure fluctuations on steps immersed in turbulent boundary layers," J. Fuild Mech., Vol. 712, 2012, pp. 471-504. doi:10.1017/jfm.2012.433. 